\title{
High-frequency waves in the solar coronal plasma ${ }^{\star}$
}

\author{
V. G. Ledenev ${ }^{1}$, V. V. Tirsky ${ }^{2}$, and V. M. Tomozov ${ }^{1}$ \\ 1 Institute of Solar-Terrestrial Physics, Russian Academy of Sciences, PO Box 4026, Irkutsk, Russia \\ e-mail: leden@iszf.irk.ru \\ 2 Institute of Laser Physics, Russian Academy of Sciences, Irkutsk Branch, Russia
}

Received 4 July 2005 / Accepted 8 February 2006

\begin{abstract}
We derived numerical solutions of a dispersion equation in order to analyze the effect of finite plasma temperature on the highfrequency wave dispersion characteristics in conditions of hot magnetized plasma in the solar corona. Spectra of the high-frequency eigen modes of these plasma were determined in conditions when the electron gyrofrequency is lower than the plasma one and when the eigen modes frequencies are higher than the electron gyrofrequency. The longitudinal wave mode is shown to turn to the Z-mode at refractive index $n<1$. At refractive index $n \gg 1$, the longitudinal wave frequency increases when $n$ grows, and these waves go to strongly damped ones with an anomalous dispersion. We interpret some spectral features of type II and IV radio bursts in the solar corona.
\end{abstract}

Key words. Sun: activity - Sun: corona - plasmas

\section{Introduction}

The solar corona is a high-temperature $\left(>10^{6} \mathrm{~K}\right)$ plasma that is filled with magnetic fields with structural forms on different scales. Numerous processes of energy release take place in the dynamical solar coronal plasma. These events cover wide spatial and temporal ranges (mainly in the form of nanoflares, microflares, flares, and coronal mass ejections), which are connected to magnetic field energy dissipation processes. The occurrence of fast plasma flows, beams of energetic particles, and shock waves accompany these processes. In turn, these energy release channels are connected with the appearance of radio emission in the form of different types of radio emission bursts owing to the development of plasma instabilities (Zheleznyakov 1970; Kaplan \& Tsytovich 1973; Melrose 1980; Altyntsev et al. 1982). Similar bursts of much more intensive radio emission are observed in stars of late spectral classes (e.g., red dwarfs and close binary systems of F, G, K, M spectral classes) with high flare activity and high-temperature stretching coronae.

It is known that the analogy between the Sun and these classes of stars is usually used quite successfully to explain radio emission bursts from such stars (Gershberg \& Pikelner 1972; Gershberg 2002; Stepanov 2003). Investigating the physical mechanisms of the radio emission generation in solar and stellar coronae is quite a significant problem for the diagnostics of the physical parameters of the coronal plasma. Though the energy release portion related to radio emission in the solar corona is relatively small, the physical parameters in the coronal plasma can be estimated from the basic characteristics of the radio emission (brightness temperature, polarization, radiation pulsations, and frequency drifts).

In our opinion, it is necessary to pay special attention to radio emission bursts with fine structure, because such burst structures are directly related to the radiation mechanism. A large number

\footnotetext{
* Appendix A is only available in electronic form at
} http://www.edpsciences.org of varied solar and stellar radio emission bursts were interpreted on the basis of the plasma mechanism, including the excitation of high-frequency plasma waves by fast electron beams, with subsequent conversion of these waves into electromagnetic radiation. Besides, the plasma radiation mechanisms in coronal magnetic fields can be related to the instabilities of fast particle distribution functions in magnetic traps with a loss cone. These plasma instabilities are able to generate small-scale waves of different types, e.g., cyclotron harmonic waves, whistlers, and Langmuir waves.

In addition, (apart from the mechanisms mentioned above) to explain narrow band $(\Delta \omega / \omega \approx 0.01-0.1, \omega$ is emission frequency), short-term (10-100 ms) solar events (radio spikes) in the coronal plasma of the Sun, the electron-cyclotron maser mechanism is used successfully (Melrose \& Dulk 1982; Fleishman \& Melnikov 1998), and this mechanism leads to different types of difficulty in high-temperature stellar coronae: anomalously high Alfven velocities, the outcome problem of radio emission, etc. (Stepanov 2003).

Until now, interpretation of solar radio emission has been based on the spectra of eigen oscillations in approximating either isotropic or cold magnetized plasma. Usually, it is assumed that the effect of a plasma finite temperature could lead to some small corrections in results. Indeed, in many cases this approximation does lead to correct results, such as in the particular case of wave propagation at small angles to the magnetic field. For example, such a situation is realized in type III bursts when the energetic electron beam, which excites a beam instability, moves along the coronal magnetic field, and the direction to the observer coincides with a course of electron-beam motion. In this case, dispersion characteristics of transverse electromagnetic waves coincide with characteristics of the waves in the magnetized plasma.

However, this situation changes if the radiation is observed at an angle to a magnetic field. As angle $\theta$ between the magnetic field and wave propagation direction is not equal to zero, the dispersion curves for ordinary and extraordinary waves 
approaching each other, and they almost merge in the weak magnetic field. The calculations that were made in our paper show that, for longitudinal waves with refractive indexes $n>10$, dispersion characteristics change substantially. In this case, the effect of even a relatively weak magnetic field $\left(\omega_{\mathrm{He}}^{2} \ll \omega_{\mathrm{pe}}^{2}\right.$, where $\omega_{\mathrm{He}}$ is the electron cyclotron frequency and $\omega_{\mathrm{pe}}$ the electron plasma frequency) essentially changes the dispersion characteristics of high-temperature plasma. In particular, the longitudinal wave frequency increases as the refractive index grows, and a branch appears corresponding to waves with negative (anomalous) dispersion, e.g. the waves whose frequency decreases as the wave number increases. Besides, the longitudinal waves go to the Z-mode with the refractive index $n<1$, as it propagates at an angle to the magnetic field. This mode shifts into the frequency region lower than $\omega_{\mathrm{pe}}$. Since the solar corona is a hot plasma with a magnetic field, these results have to be taken into account in many of the present and future models of sporadic radio emissions of the Sun.

A considerable amount of papers and books are focussed on the study of different types of high-frequency waves in the magnetized plasma (see, e.g., Baldwin et al. 1969; Ginzburg 1970; Akhiezer et al. 1975; Ginzburg \& Rukhadze 1975; Melrose 1980). For instance, the particular cases of wave propagation along and across the magnetic field were considered in detail. In a review paper by Baldwin et al. (1969), the dispersion equations for transverse (electromagnetic) and longitudinal (plasma) waves are quoted, which were derived in the approximation of the weak magnetic field and relatively low temperature. From these equations one can conclude that the characteristics of eigen plasma oscillations change even in this approximation. The finite temperature plasma effect is considered on the basis of a quasi-hydrodynamic approximation (Ginzburg 1970), but this approach does not allow the kinetic effects to be taken into account completely, although they do influence the eigen oscillation parameters considerably. In Ginzburg (1970) the kinetic approach was realized in a frequency range close to the electron plasma frequency without taking terms in the dispersion equation into account that are proportional to $\left(v_{\mathrm{Te}} / c\right)^{2}$ (where $v_{\mathrm{Te}}$ is the thermal velocity of electrons) and without the effects of cyclotron harmonics above the second one. Willes \& Cairns (2000) obtained an approximate solution for the dispersion equation of a magnetized kinetic plasma for small $\theta$ by using an expansion of the dielectric tensor longitudinal component in terms of the parameter $\rho^{2}=\left(k v_{\mathrm{Te}} / \omega_{\mathrm{He}}\right)^{2}$. However, the cases under consideration do not give a full notion of the eigen oscillation characteristics of a high-temperature magnetized plasma, which can be used for correctly interpreting the characteristics of radio emission bursts in solar and stellar coronae. To obtain these characteristics, an investigation of a full dispersion equation is necessary. The solution of this equation can only be obtained by numerical methods.

A paper by Andre (1985) and a review by Rönnmark (1985) both show the dispersion surfaces for high-frequency plasma waves taking the effect of finite temperature plasma into account, which were determined by numerical methods. These results, however, were obtained for parameters that are typical of the Earth's magnetosphere and are essentially different from solar corona parameters. Furthermore, these results were obtained with highly simplifying assumptions in the numerical computation procedure.

In the present paper we investigate different types of highfrequency waves in a high-temperature magnetized plasma at $\omega_{\mathrm{He}} / \omega_{\mathrm{pe}}<1$ on the basis of a numerical solution of the dispersion equation. In Sect. 2 the dispersion equation describing high-frequency waves in a high-temperature magnetized plasma is presented in its most general form, but unwieldy formulae are moved into the appendix. This section describes the results of a numerical solution of the dispersion equation in detail and presents the high-frequency wave characteristics by taking into account their propagation at different angles to the magnetic field. On the basis of results for the characteristics of highfrequency waves in a high-temperature plasma, we provide a qualitative interpretation of some features of type II and IV bursts in Sect. 3. In the conclusion, the main results of this work are reported, followed by a brief discussion.

\section{Characteristics of high-frequency waves in hot magnetized plasma}

To provide an overall picture of the eigen oscillation characteristics of a hot magnetized plasma, it is necessary to investigate a complete dispersion equation of such oscillations in the form (Akhiezer et al. 1975; Ginzburg \& Rukhadze 1975):

$a+\left(\omega^{2} /(k c)^{2}\right) b+\left(\omega^{4} /(k c)^{4}\right) c_{0}=0$

where $a=\left(k_{\perp}^{2} / k^{2}\right) \varepsilon_{x x}+\left(k_{z}^{2} / k^{2}\right) \varepsilon_{z z}+2\left(k_{\perp} k_{z} / k^{2}\right) \varepsilon_{x z}, b=$ $-\varepsilon_{x x} \varepsilon_{z z}+\varepsilon_{x z}^{2}-\left(k_{z} / k\right)^{2}\left(\varepsilon_{y y} \varepsilon_{z z}+\varepsilon_{y z}^{2}\right)-\left(k_{\perp} / k\right)^{2}\left(\varepsilon_{x x} \varepsilon_{y y}+\varepsilon_{x y}^{2}\right)+$ $2\left(k_{\perp} k_{z} / k^{2}\right)\left(\varepsilon_{x y} \varepsilon_{y z}-\varepsilon_{x z} \varepsilon_{y y}\right), c_{0}=\varepsilon_{z z}\left(\varepsilon_{x x} \varepsilon_{y y}+\varepsilon_{x y}^{2}\right)+\varepsilon_{x x} \varepsilon_{y z}^{2}-\varepsilon_{y y} \varepsilon_{x z}^{2}+$ $2 \varepsilon_{y z} \varepsilon_{x z} \varepsilon_{x y}$.

Here, $\omega$ is the complex frequency, $k$ the wave number, $k_{z}$ the longitudinal (with respect to the magnetic field) wave vector component, $k_{\perp}$ the transverse wavevector component, and $\varepsilon_{i j}$ stands for the components of the dielectric tensor.

The waves in magnetized plasma, for which the refractive index $n^{2}=k^{2} c^{2} / \omega^{2} \gg 1$, are customarily considered as longitudinal (Ginzburg \& Rukhadze 1975). It is assumed that their dispersion properties are determined by the first term of Eq. (1). But it is correct for those values of the refractive index that are high enough. For the oscillations with refractive indices $n \leq 1$, it is difficult to define the mode of the high-frequency wave as purely transverse or purely longitudinal, so the contribution from the second and third terms to the solution of Eq. (1) cannot be neglected any longer. As shown below, taking the second and third terms in Eq. (1) for $n \leq 1$ into account leads to a substantial change in the dispersion characteristics of the waves in a hot magnetized plasma as compared to the cold one.

Figures 1a-d present the results of calculations (by formulas A.1) for the dependence of the refractive index $n$ on the frequency of weakly damped modes for angles between the wave vector and the magnetic field $\theta=0.0$ and 1.0 for the parameters $\eta=\omega_{\mathrm{pe}} / \omega_{\mathrm{He}}=5$ and 10 , and $v_{\mathrm{Te}}^{2} / c^{2}=2 \times 10^{-4}$. These values are consistent with the parameters of the solar corona. The summation over cyclotron harmonics was performed up to the fifteenth one. The contribution from higher harmonics does not alter the character of the dispersion curves for the waves substantially. Landau-damping is not significant for $\omega / k \gg v_{\mathrm{Te}}$, which means that the refractive index of weakly damped waves is $n \ll 70$ in this case. We also considered the waves, which obey weaker inequality $n<70$, since these waves can grow if the growth rate exceeds the Landau-damping rate. As in the case of quasihydrodynamic approximation (Ginzburg 1970), here we have three high-frequency branches of waves. In the case of the propagation at small angles to the magnetic field (Figs. 1a and 1c), the waves may be identified as two transverse waves - the extraordinary wave or X-mode (branch 1 ), and the ordinary wave or O-mode (branch 2) - and as the longitudinal wave (branch 3), 

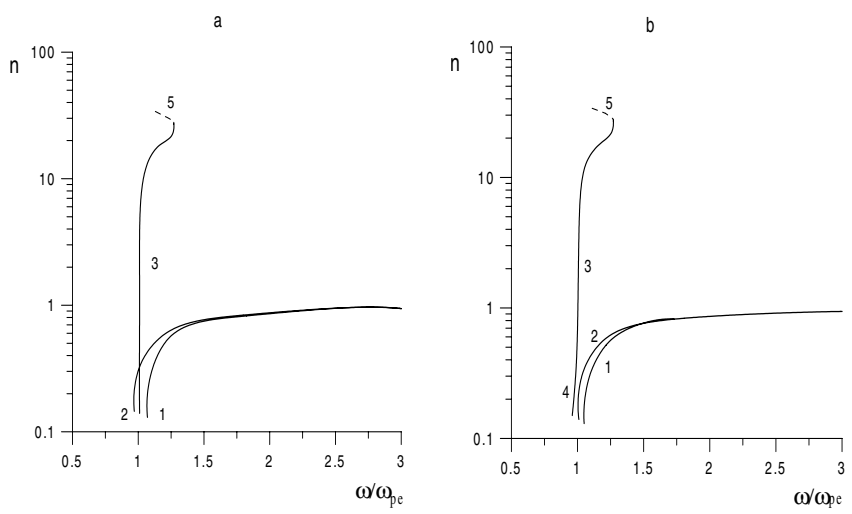

$c$
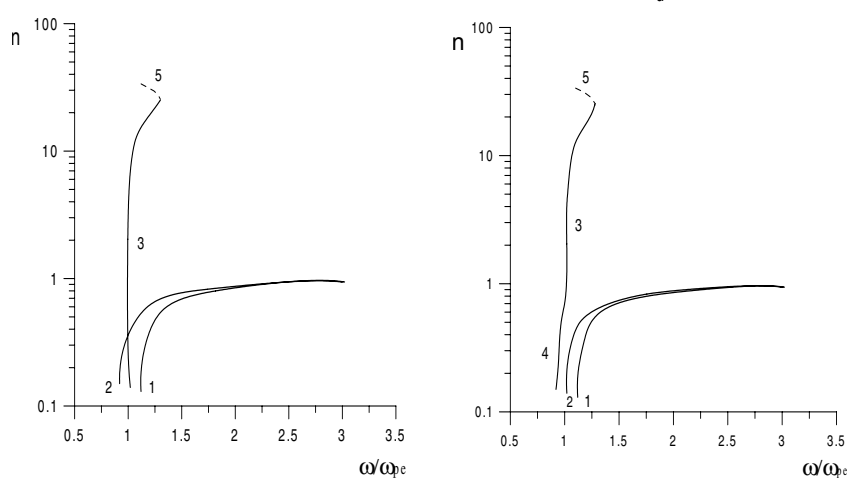

Fig. 1. Frequency dependence of the refractive index for $\omega_{\mathrm{pe}} / \omega_{\mathrm{He}}=10$ the angle between the wave vector and magnetic field $\theta=0.0$ (panel a)), $\theta=1.0$ (panel b)), and for $\omega_{\mathrm{pe}} / \omega_{\mathrm{He}}=5, \theta=0.0$ (panel c) $), \theta=1.0$ (panel d)). Branches 1, 2, and 3 correspond to the X (extraordinary), $\mathrm{O}$ (ordinary), and longitudinal modes, respectively. Branch 4 corresponds to the Z-mode, branch 5 corresponds to the waves with anomalous dispersion. $v_{\mathrm{Te}}^{2} / c^{2}=2 \times 10^{-4}$.

which turns to the Z-mode for $n \leq 1$ (branch 4) (Ginzburg 1970; Melrose 1980).

The case of strict wave propagation along the magnetic field is a degenerated one, in the sense that the solutions of the dispersion equation for longitudinal and transverse waves are independent. In this case longitudinal waves are described by a well-known dispersion relation (Akhiezer et al. 1975; Ginzburg \& Ruchadze 1975) that is defined by the equality to zero of the first term of Eq. (1) or via $\operatorname{Re}\{a\}=0$ (see A.1). At $\theta=0$ there is an intersection of longitudinal and ordinary transverse wave branches (Figs. 1a and 1c) and a transition of one type of wave into another. However, at $\theta \neq 0$ longitudinal wave turns to Z-mode in the region $n<1$, and the Z-mode branch goes to the frequency region lower than the plasma one. This result agrees with Willes \& Cairns (2000), who have found an approximate solution of the dispersion equation for the Maxwellian magnetized plasma by means of the expansion in terms of the parameter $\left(k v_{\mathrm{Te}} / \omega_{\mathrm{He}}\right)^{2}$. Cutoff frequencies for ordinary and extraordinary waves are determined by the same relations as in the cold magnetized plasma (Ginzburg 1970). As one can see from Figs. 1a-d, the distance between the cutoff frequencies increases when the magnetic field grows. As the angle $\theta \neq 0$, the cutoff frequencies approach each other and the frequency for ordinary waves shifts into the frequency region higher than $\omega_{\text {pe }}$.

The most essential features of longitudinal wave-dispersion characteristics become apparent for refractive index $n>10$ (Figs. 1a-d). As $n$ grows in this region, the longitudinal wavefrequency increases and reaches the maximum value $\omega \sim 1.3 \omega_{\text {pe }}$

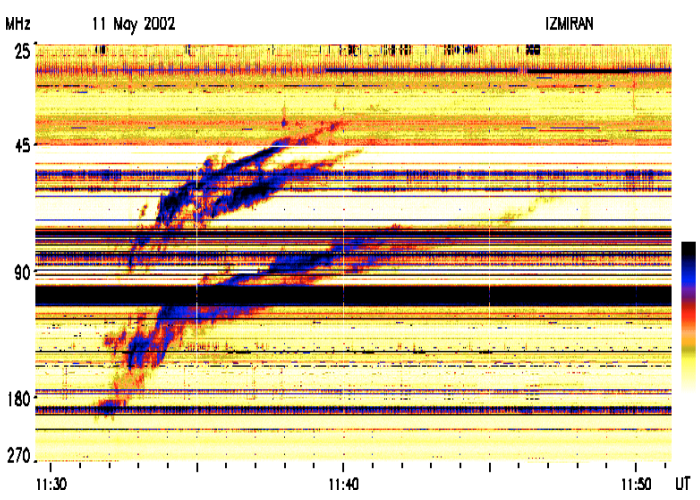

Fig. 2. Type II solar radio burst with harmonic-component splitting.

at $n \sim 30$. At the same time, the Landau damping grows, since the wave phase velocity approaches the electron thermal velocity. At $n \sim 30$ and $\omega \sim 1.3 \omega_{\mathrm{pe}}$, the damping achieves a value of about $0.1 \omega$. Such waves can be excited only by strong instability. As the refractive index rises further, the longitudinal wave frequency goes down, and the branch of anomalous dispersion waves appears, i.e. the waves whose frequency decreases as the wave number increases. In Figs. 1a-d this branch is marked by dotted lines. However, these waves strongly damp because, as the refractive index grows, the wave phase velocity becomes equal to the electron thermal velocity.

The growth of the longitudinal wave frequency in the region $n \geq 10$ is caused by the finite cyclotron radius effect. This is a consequence of the fact that this growth is observed when the parameter $k v_{\mathrm{Te}} / \omega_{\mathrm{He}}$ achieves unity in order of magnitude; i.e. the wave length is close to the cyclotron radius.

Our calculations show the weak angular dependence of the hot-plasma dispersion characteristics for longitudinal waves. However, as $\theta$ approaches $90^{\circ}(\theta \sim 1.4-1.5$ radian $)$, the Bernstein longitudinal modes become apparent in the spectrum of weakly damped plasma oscillations at the frequencies close to the cyclotron harmonic frequencies. It is difficult to calculate the spectra in this case.

\section{Interpreting some features of solar radio bursts}

The results allow us to explain some features of radio emissions of type II and IV bursts from a unified point of view and to obtain more accurate estimations of magnetic field strength in the solar coronal plasma.

The characteristic feature of the type II bursts is the harmonic component splitting into two bands (Fig. 2) with the distance between them about 10-20\% of the emission frequency (Kundu 1965; Zheleznyakov 1970). Ledenev \& Urbarz (1993) explained the type II harmonic structure by the emission generation at the frequencies corresponding to the cyclotron resonance $\omega-k_{\|} v_{\|}-s \omega_{\mathrm{He}}=0$, where $\omega$ is the frequency of longitudinal waves that are excited by the beam-anisotropic instability (Mikhailovsky 1974), $k_{\|}$is the longitudinal (with respect to the magnetic field) component of the wave vector, $v_{\|}$the longitudinal component of the electron velocity, and s the number of the cyclotron harmonic. However, there was no convincing explanation of the origin of the harmonic component splitting, while, as a rule, no more than two splitting bands are observed. Our calculations allow us to give a more natural explanation for this event. As one can see from Figs. 1a-d, the frequency band of the waves excited at high values of the refractive index (i.e. at the lowest energies of electrons, which excite longitudinal waves) 
can achieve $30 \%$. If the electron cyclotron frequency is about $10 \%$ of the emission frequency, no more than two cyclotron harmonics will most likely fall into the band of longitudinal wave generation. At that point one should take into account that the distance between the splitting bands, generally speaking, is not equal to the cyclotron frequency, because the resonance regions at the adjacent cyclotron harmonics are shifted in the height due to the inhomogeneity of the solar corona. Different longitudinal wave frequencies and cyclotron frequencies correspond to this resonance. Nevertheless, if the condition $k_{\|} v_{\|} \ll \omega_{\mathrm{He}}$ is satisfied, the distance between the splitting bands can be used for estimating the magnetic field in the region of type-II-burst generation. This condition is satisfied, if the emission is generated at angles that are large enough to the magnetic field. Such estimation for the event in Fig. 2 is about $10 \mathrm{GHz}$ for the splitting frequency, which corresponds to $6 \mathrm{G}$ for the magnetic field.

The longitudinal wave excitation in a wide-frequency band allow us to explain the nature of the type IV wide-band bursts (Kundu 1965; Zheleznyakov 1970) in greater detail. It has been suggested that type IV bursts are generated by the plasma mechanism (Stepanov 1973; Ledenev 1982), and that wide-band emission is due to the inhomogeneity of the coronal density. Our calculations show that the wide-band emission can be implemented without taking this coronal density inhomogeneity into account. The wideband emission is generated due to the excitation of longitudinal waves through the beam instability in the wide frequency band (up to $30 \%$ at the fixed level in the corona). Though these waves strongly damp, electron beams with the velocities about the thermal one can be so powerful that the wave's growth rate will be higher than the damping rate at the background electrons. When taking the corona inhomogeneity and the electron velocity spread into account, the emission band can achieve almost $100 \%$.

As is known, the sources of type III bursts (electron beams) move along the coronal magnetic field (Zheleznyakov \& Zaitsev 1970; Dulk \& Suzuki 1980). Ledenev \& Messerotti (1999) suggested estimating the magnetic field in the corona by using the type III burst polarization. These bursts often show high polarization at the beginning of the burst. It was suggested that this effect is caused by generation of the emission in the region where only ordinary waves propagate. The frequency distance between the cutoff frequencies of ordinary and extraordinary waves determines the frequency bandwidth where high polarization is observed. In turn, the frequency bandwidth is proportional to the magnetic field. Our calculations show that the cutoff frequencies of these waves do not change. At this point, the magnetic field estimation offered by Ledenev \& Messerotti (1999) can be used in the hot plasma of the solar corona.

\section{Conclusion}

We investigated the dispersion characteristics of weakly damped, high-frequency waves in a high-temperature magnetized plasma on the basis of a numerical solution of the dispersion equation. Our results show that the dispersion characteristics of the hot magnetized plasma have the following features compared with the cold magnetized plasma.

1. Dispersion characteristics of ordinary and extraordinary waves differ significantly when propagating along the magnetic field. The dispersion curves of these waves approach each other when the angle between the wave vector and the magnetic field $\neq 0$.

2. The longitudinal mode turns into the $Z$-mode in the region of the refractive index $n<1$. The Z-mode frequency shifts in the frequency region lower that is the plasma one.

3. The longitudinal wave frequency increases at the refractive indexes $n>10$ up to the value about $1.3 \omega_{\text {pe }}$ when $n$ rises. The wave branch with anomalous dispersion appears with the further refractive index increase. The frequency of these waves decreases when the wave number increases. However, this branch strongly damps due to Landau-damping.

Our calculations allow us to explain some observational features of solar radio emission bursts. Emission generation is the most effective at the cyclotron resonance. Splitting of the type II burst harmonic components is explained by the fact that no more than two frequencies corresponding to the cyclotron resonance condition fall into the emission generation range. The generation of the wide-band emission continua (IV type bursts) is explained by the longitudinal wave excitation at the wide frequency band (up to $30 \%$ at the fixed level in the corona).

It should be noted, moreover, that these effects must be appearing more distinctly in the characteristics of the radio emission of late spectral class stars owing to the high temperature of the star's coronae, but more specific investigations are still needed.

Acknowledgements. This work was carried out with support of RFBR grant 0302-16229, Program of basic research RAS N30, and the Scientific School grants 477.2003.2 and RI-112/001/045. We acknowledge the IZMIRAN solar radio group who kindly provided the spectrographic radio data. The authors express appreciation to the referee for useful remarks and corrections that assisted them in improving the paper.

\section{References}

Abramovitz, M., \& Stegun, I. (ed.) 1964, Handbook of Mathematical Functions, Nat. Bureau of Standards, N-Y, 832

Altyntsev, A. T., Banin, V. G., Kuklin, G. V., \& Tomozov, V. M. 1982, Solar Flares (Nauka), 246

Akhiezer, A. I., Akhiezer, I. A., Polovin, R. V., Sitenko, A. G., \& Stepanov, K. N. 1975, Plasma Electrodynamics (Oxford: Pergamon Press), 720

Andre, M. 1985, J. Plas. Phys., 33, 1

Baldvin, D. E., Bernstein, I. B., \& Veenink, M. P. H. 1969, Adv. Plasma Phys., Vols. 3, 4 (N-Y: Interscience Publ.), 172

Dulk, G. A., \& Suzuki, S. 1980, A\&A, 88, 203

Fleishman, G. D., \& Melnikov, V. F. 1998, Adv. Phys. Sci., 168, 1265

Gershberg, R. E., \& Pikelner, S. B. 1972, Comments Astrophys. Space Phys., 4, 113

Gershberg, R. E. 2002, Activity of Main Sequence Solar Type Stars, Odessa, Astroprint, 688

Ginzburg, V. L. 1970, The Propagation of Electromagnetic Waves in Plasmas (Oxford: Pergamon Press), 684

Ginzburg, V. L., \& Rukhadze, A. A. 1975, Waves in Magnetoactive Plasma (Nauka), 256

Kaplan, S. A., \& Tsytovich, V. N. 1973, Plasma Astrophysics (Oxford: Pergamon Press), 440

Kundu, M. R. 1965, Solar Radio Astronomy (N-Y: Interscience Publ.), 660

Ledenev, V. G. 1982, Soviet Astron., 26, 452

Ledenev, V. G., \& Urbarz, H. 1993, Sol. Phys., 146, 365

Ledenev, V. G., \& Messerotti, M. 1999, Sol. Phys., 185, 143

Melrose, D. B. 1980, Plasma Astrophysics (N-Y: Gordon and Breach), Vol. 1, $269,2,423$

Melrose, D. B., \& Dulk, G. A. 1982, ApJ, 259, 844

Mikhailovsky, A. B. 1974, Theory of Plasma Instabilities (N-Y: Consultant Bureau), Vol. 1, 272

Rönnmark, K.-G. 1985, Space Sci. Rev., 42, 411

Stepanov, A. V. 1973, Soviet Astron., 17, 781

Stepanov, A. V. 2003, Adv. Phys. Sci., 173, 106

Willes, A. J., \& Cairns, I. H. 2000, Phys. Plasm., 7, 3167 
Zheleznyakov, V. V. 1970, Radio Emission from the Sun and Planets (Oxford: Pergamon Press), 560

Zheleznyakov, V. V., \& Zaitsev, V. V. 1970, Sov. Astron. - AZh, 14, 47 
V. G. Ledenev et al.: High-frequency waves in the solar coronal plasma, Online Material $p 1$

\section{Online Material}




\section{Appendix}

The dispersion Eq. (1) for waves in a hot magnetized plasma may be represented as

$$
\begin{aligned}
\operatorname{Re}\left\{a+\frac{\omega^{2}}{k^{2} c^{2}} b+\frac{\omega^{4}}{k^{4} c^{4}} c_{0}\right\}= & \operatorname{Re}\{a\}+\frac{\left(\Omega^{2}-\Gamma^{2}\right) v_{\mathrm{Te}}^{2}}{\rho^{2} c^{2}}\left[\xi_{1}-\operatorname{Re}\{a\} \operatorname{Re}\left\{S p \varepsilon_{i j}\right\}+\operatorname{Im}\{a\} \operatorname{Im}\left\{S p \varepsilon_{i j}\right\}\right] \\
& -\frac{2 \Omega \Gamma v_{\mathrm{Te}}^{2}}{\rho^{2} c^{2}}\left[\xi_{2}-\operatorname{Re}\{a\} \operatorname{Im}\left\{S p \varepsilon_{i j}\right\}-\operatorname{Im}\{a\} \operatorname{Re}\left\{S p \varepsilon_{i j}\right\}\right]+\operatorname{Re}\left\{c_{0} \frac{\omega^{4}}{k^{4} c^{4}}\right\}=0, \\
\operatorname{Im}\left\{a+\frac{\omega^{2}}{k^{2} c^{2}} b+\frac{\omega^{4}}{k^{4} c^{4}} c_{0}\right\}= & \operatorname{Im}\{a\}+\frac{(2 \Omega \Gamma) v_{\mathrm{Te}}^{2}}{\rho^{2} c^{2}}\left[\xi_{1}-\operatorname{Re}\{a\} \operatorname{Re}\left\{S p \varepsilon_{i j}\right\}+\operatorname{Im}\{a\} \operatorname{Im}\left\{S p \varepsilon_{i j}\right\}\right] \\
& +\frac{\left(\Omega^{2}-\Gamma^{2}\right) v_{\mathrm{Te}}^{2}}{\rho^{2} c^{2}}\left[\xi_{2}-\operatorname{Re}\{a\} \operatorname{Im}\left\{S p \varepsilon_{i j}\right\}-\operatorname{Im}\{a\} \operatorname{Re}\left\{S p \varepsilon_{i j}\right\}+\operatorname{Im}\left\{c_{0} \frac{\omega^{4}}{\left.k^{4} c^{4}\right\}=0 .}\right.\right.
\end{aligned}
$$

Here, $\gamma$ is the wave damping coefficient, $v_{\mathrm{Te}}$ the thermal velocity of plasma electrons, $\Omega=\omega / \omega_{\mathrm{He}}, \Gamma=\gamma / \omega_{\mathrm{He}}, \eta=$ $\omega_{\mathrm{pe}} / \omega_{\mathrm{He}}, \rho=k \mathrm{v}_{\mathrm{Te}} / \omega_{\mathrm{He}}, \operatorname{Re}\{a\}=1+\frac{\eta^{2}}{\rho^{2}}-(\pi / 2)^{1 / 2} \frac{\eta^{2}}{\rho^{3} \cos \theta} \sum_{-\infty}^{\infty} A_{s}\{\Gamma \operatorname{Re} W+\Omega \operatorname{Im} W\}, \operatorname{Im}\{a\}=-(\pi / 2)^{1 / 2} \frac{\eta^{2}}{\rho^{3} \cos \theta} \sum_{s=-\infty}^{\infty} A_{s}\{\Gamma \operatorname{Im} W-$ $\Omega \operatorname{Re} W\}, A_{s}\left(\rho^{2} \sin ^{2} \theta\right)=I_{s}\left(\rho^{2} \sin ^{2} \theta\right) \exp \left(-\rho^{2} \sin ^{2} \theta\right)$, and $I_{s}$ is a modified Bessel function,

$$
\begin{aligned}
\xi_{1}=\operatorname{Re}\left\{\frac{k_{i} k_{j} \varepsilon_{i \mu} \varepsilon_{\mu j}}{k^{2}}\right\}= & \left(\sin \theta \operatorname{Re}\left\{\varepsilon_{x x}\right\}+\cos \theta \operatorname{Re}\left\{\varepsilon_{x z}\right\}\right)^{2}-\left(\sin \theta \operatorname{Im}\left\{\varepsilon_{x x}\right\}+\cos \theta \operatorname{Im}\left\{\varepsilon_{x z}\right\}\right)^{2}-\left(\sin \theta \operatorname{Re}\left\{\varepsilon_{x y}\right\}-\cos \theta \operatorname{Re}\left\{\varepsilon_{y z}\right\}\right)^{2} \\
& +\left(\sin \theta \operatorname{Im}\left\{\varepsilon_{x y}\right\}-\cos \theta \operatorname{Im}\left\{\varepsilon_{y z}\right\}\right)^{2}+\left(\sin \theta \operatorname{Re}\left\{\varepsilon_{x z}\right\}+\cos \theta \operatorname{Re}\left\{\varepsilon_{z z}\right\}\right)^{2}-\left(\sin \theta \operatorname{Im}\left\{\varepsilon_{x z}\right\}+\cos \theta \operatorname{Im}\left\{\varepsilon_{z z}\right\}\right)^{2}
\end{aligned}
$$

$$
\begin{aligned}
\xi_{2}=\operatorname{Im}\left\{\frac{k_{i} k_{j} \varepsilon_{i \mu} \varepsilon_{\mu j}}{k^{2}}\right\}= & 2\left(\sin \theta \operatorname{Re}\left\{\varepsilon_{x x}\right\}+\cos \theta \operatorname{Re}\left\{\varepsilon_{x z}\right\}\right)\left(\sin \theta \operatorname{Im}\left\{\varepsilon_{x x}\right\}+\cos \theta \operatorname{Im}\left\{\varepsilon_{x z}\right\}\right)-2\left(\sin \theta \operatorname{Re}\left\{\varepsilon_{x y}\right\}\right. \\
& \left.-\cos \theta \operatorname{Re}\left\{\varepsilon_{y z}\right\}\right)\left(\sin \theta \operatorname{Im}\left\{\varepsilon_{x y}\right\}-\cos \theta \operatorname{Im}\left\{\varepsilon_{y z}\right\}\right)+2\left(\sin \theta \operatorname{Re}\left\{\varepsilon_{x z}\right\}+\cos \theta \operatorname{Re}\left\{\varepsilon_{z z}\right\}\right)\left(\sin \theta \operatorname{Im}\left\{\varepsilon_{x z}\right\}\right. \\
& \left.+\cos \theta \operatorname{Im}\left\{\varepsilon_{z z}\right\}\right),
\end{aligned}
$$

$\operatorname{Re}\left\{S p \varepsilon_{i j}\right\}=\operatorname{Re}\left\{\varepsilon_{x x}+\varepsilon_{y y}+\varepsilon_{z z}\right\}, \quad \operatorname{Im}\left\{S p \varepsilon_{i j}\right\}=\operatorname{Im}\left\{\varepsilon_{x x}+\varepsilon_{y y}+\varepsilon_{z z}\right\}$

$\operatorname{Re}\left\{c_{0} \frac{\omega^{4}}{k^{4} c^{4}}\right\}=\frac{v_{\mathrm{Te}}^{4}}{\rho^{4} c^{4}}\left\{\operatorname{Re}\left\{c_{0}\right\}\left[\left(\Omega^{2}-\Gamma^{2}\right)^{2}-4 \Omega^{2} \Gamma^{2}\right]-\operatorname{Im}\left\{c_{0}\right\} 4 \Omega \Gamma\left(\Omega^{2}-\Gamma^{2}\right)\right\}$,

$\operatorname{Im}\left\{c_{0} \frac{\omega^{4}}{k^{4} c^{4}}\right\}=\frac{v_{\mathrm{Te}}^{4}}{\rho^{4} c^{4}}\left\{\operatorname{Im}\left\{c_{0}\right\}\left[\left(\Omega^{2}-\Gamma^{2}\right)^{2}-4 \Omega^{2} \Gamma^{2}\right]+\operatorname{Re}\left\{c_{0}\right\} 4 \Omega \Gamma\left(\Omega^{2}-\Gamma^{2}\right)\right\}$,

$\operatorname{Re}\left\{c_{0}\right\}=\left[\operatorname{Re}\left\{\varepsilon_{z z}\right\} \operatorname{Re}\left\{\varepsilon_{x x}\right\}-\operatorname{Im}\left\{\varepsilon_{z z}\right\} \operatorname{Im}\left\{\varepsilon_{x x}\right\}\right] \operatorname{Re}\left\{\varepsilon_{y y}\right\}-\left[\operatorname{Re}\left\{\varepsilon_{z z}\right\} \operatorname{Im}\left\{\varepsilon_{x x}\right\}+\operatorname{Im}\left\{\varepsilon_{z z}\right\} \operatorname{Re}\left\{\varepsilon_{x x}\right\}\right] \operatorname{Im}\left\{\varepsilon_{y y}\right\}+\left[\left(\operatorname{Re}\left\{\varepsilon_{x y}\right\}\right)^{2}-\left(\operatorname{Im}\left\{\varepsilon_{x y}\right\}\right)^{2}\right] \operatorname{Re}\left\{\varepsilon_{z z}\right\}$ $-2 \operatorname{Re}\left\{\varepsilon_{x y}\right\} \operatorname{Im}\left\{\varepsilon_{x y}\right\} \operatorname{Im}\left\{\varepsilon_{z z}\right\}+\left[\left(\operatorname{Re}\left\{\varepsilon_{y z}\right\}\right)^{2}-\left(\operatorname{Im}\left\{\varepsilon_{y z}\right\}\right)^{2}\right] \operatorname{Re}\left\{\varepsilon_{x x}\right\}-2 \operatorname{Re}\left\{\varepsilon_{y z}\right\} \operatorname{Im}\left\{\varepsilon_{y z}\right\} \operatorname{Im}\left\{\varepsilon_{x x}\right\}-\left[\left(\operatorname{Re}\left\{\varepsilon_{x z}\right\}\right)^{2}-\left(\operatorname{Im}\left\{\varepsilon_{x z}\right\}\right)^{2}\right] \operatorname{Re}\left\{\varepsilon_{y y}\right\}$ $+2 \operatorname{Re}\left\{\varepsilon_{x z}\right\} \operatorname{Im}\left\{\varepsilon_{x z}\right\} \operatorname{Im}\left\{\varepsilon_{y y}\right\}+2\left[\operatorname{Re}\left\{\varepsilon_{x y}\right\} \operatorname{Re}\left\{\varepsilon_{x z}\right\}-\operatorname{Im}\left\{\varepsilon_{x y}\right\} \operatorname{Im}\left\{\varepsilon_{x z}\right\}\right] \operatorname{Re}\left\{\varepsilon_{y z}\right\}-2\left[\operatorname{Re}\left\{\varepsilon_{x y}\right\} \operatorname{Im}\left\{\varepsilon_{x z}\right\}+\operatorname{Im}\left\{\varepsilon_{x y}\right\} \operatorname{Re}\left\{\varepsilon_{x z}\right\}\right] \operatorname{Im}\left\{\varepsilon_{y z}\right\}$,

$\operatorname{Im}\left\{c_{0}\right\}=\left[\operatorname{Re}\left\{\varepsilon_{z z}\right\} \operatorname{Im}\left\{\varepsilon_{x x}\right\}+\operatorname{Im}\left\{\varepsilon_{z z}\right\} \operatorname{Re}\left\{\varepsilon_{x x}\right\}\right] \operatorname{Re}\left\{\varepsilon_{y y}\right\}+\left[\operatorname{Re}\left\{\varepsilon_{z z}\right\} \operatorname{Re}\left\{\varepsilon_{x x}\right\}-\operatorname{Im}\left\{\varepsilon_{z z}\right\} \operatorname{Im}\left\{\varepsilon_{x x}\right\}\right] \operatorname{Im}\left\{\varepsilon_{y y}\right\}+\left[\left(\operatorname{Re}\left\{\varepsilon_{x y}\right\}\right)^{2}-\left(\operatorname{Im}\left\{\varepsilon_{x y}\right\}\right)^{2}\right] \operatorname{Im}\left\{\varepsilon_{z z}\right\}$ $+2 \operatorname{Re}\left\{\varepsilon_{x y}\right\} \operatorname{Im}\left\{\varepsilon_{x y}\right\} \operatorname{Re}\left\{\varepsilon_{z z}\right\}+\left[\left(\operatorname{Re}\left\{\varepsilon_{y z}\right\}\right)^{2}-\left(\operatorname{Im}\left\{\varepsilon_{y z}\right\}\right)^{2}\right] \operatorname{Im}\left\{\varepsilon_{x x}\right\}+2 \operatorname{Re}\left\{\varepsilon_{y z}\right\} \operatorname{Im}\left\{\varepsilon_{y z}\right\} \operatorname{Re}\left\{\varepsilon_{x x}\right\}-\left[\left(\operatorname{Re}\left\{\varepsilon_{x z}\right\}\right)^{2}-\left(\operatorname{Im}\left\{\varepsilon_{x z}\right\}\right)^{2}\right] \operatorname{Im}\left\{\varepsilon_{y y}\right\}$ $-2 \operatorname{Re}\left\{\varepsilon_{x z}\right\} \operatorname{Im}\left\{\varepsilon_{x z}\right\} \operatorname{Re}\left\{\varepsilon_{y y}\right\}+2\left[\operatorname{Re}\left\{\varepsilon_{x y}\right\} \operatorname{Re}\left\{\varepsilon_{x z}\right\}-\operatorname{Im}\left\{\varepsilon_{x y}\right\} \operatorname{Im}\left\{\varepsilon_{x z}\right\}\right] \operatorname{Im}\left\{\varepsilon_{y z}\right\}+2\left[\operatorname{Re}\left\{\varepsilon_{x y}\right\} \operatorname{Im}\left\{\varepsilon_{x z}\right\}+\operatorname{Im}\left\{\varepsilon_{x y}\right\} \operatorname{Re}\left\{\varepsilon_{x z}\right\}\right] \operatorname{Re}\left\{\varepsilon_{y z}\right\}$,

$$
\begin{aligned}
\operatorname{Re}\left\{\varepsilon_{x x}\right\} & =1+(\pi / 2)^{1 / 2} \frac{\eta^{2}\left(\Gamma \alpha_{5}-\Omega \alpha_{6}\right)}{\rho \cos \theta\left(\Omega^{2}+\Gamma^{2}\right)}, \operatorname{Im}\left\{\varepsilon_{x x}\right\}=(\pi / 2)^{1 / 2} \frac{\eta^{2}\left(\Gamma \alpha_{6}+\Omega \alpha_{5}\right)}{\rho \cos \theta\left(\Omega^{2}+\Gamma^{2}\right)}, \\
\operatorname{Re}\left\{\varepsilon_{y y}\right\} & =\operatorname{Re}\left\{\varepsilon_{x x}\right\}-(2 \pi)^{1 / 2} \frac{\eta^{2}\left(\Gamma \alpha_{1}-\Omega \alpha_{2}\right) \rho \sin ^{2} \theta}{\cos \theta\left(\Omega^{2}+\Gamma^{2}\right)}, \operatorname{Im}\left\{\varepsilon_{y y}\right\}=\operatorname{Im}\left\{\varepsilon_{x x}\right\}-(2 \pi)^{1 / 2} \frac{\eta^{2}\left(\Gamma \alpha_{2}+\Omega \alpha_{1}\right) \rho \sin ^{2} \theta}{\cos \theta\left(\Omega^{2}+\Gamma^{2}\right)}, \\
\operatorname{Re}\left\{\varepsilon_{x y}\right\} & =-(\pi / 2)^{1 / 2} \frac{\eta^{2}\left(\Omega \alpha_{7}+\Gamma \alpha_{8}\right)}{\rho \cos \theta\left(\Omega^{2}+\Gamma^{2}\right)}, \operatorname{Im}\left\{\varepsilon_{x y}\right\}=-(\pi / 2)^{1 / 2} \frac{\eta^{2}\left(\Omega \alpha_{8}-\Gamma \alpha_{7}\right)}{\rho \cos \theta\left(\Omega^{2}+\Gamma^{2}\right)}, \\
\operatorname{Re}\left\{\varepsilon_{y z}\right\} & =(\pi / 2)^{1 / 2} \frac{\eta^{2} \sin \theta}{\rho \cos ^{2} \theta}\left(\alpha_{1}-\frac{\Omega \alpha_{7}+\Gamma \alpha_{8}}{\Omega^{2}+\Gamma^{2}}\right), \operatorname{Im}\left\{\varepsilon_{y z}\right\}=(\pi / 2)^{1 / 2} \frac{\eta^{2} \sin \theta}{\rho \cos ^{2} \theta}\left(\alpha_{2}-\frac{\Omega \alpha_{8}-\Gamma \alpha_{7}}{\Omega^{2}+\Gamma^{2}}\right), \\
& \operatorname{Re}\left\{\varepsilon_{x z}\right\}=(\pi / 2)^{1 / 2} \frac{\eta^{2} \sin \theta}{\rho \cos ^{2} \theta}\left(-\alpha_{4}+\frac{\Omega \alpha_{6}-\Gamma \alpha_{5}}{\Omega^{2}+\Gamma^{2}}\right), \operatorname{Im}\left\{\varepsilon_{x z}\right\}=(\pi / 2)^{1 / 2} \frac{\eta^{2} \sin \theta}{\rho \cos ^{2} \theta}\left(\alpha_{3}-\frac{\Omega \alpha_{5}+\Gamma \alpha_{6}}{\Omega^{2}+\Gamma^{2}}\right), \\
& \operatorname{Re}\left\{\varepsilon_{z z}\right\}=1+\frac{\operatorname{Re}\{a\}-1}{\cos ^{2} \theta}+(\pi / 2)^{1 / 2} \frac{\eta^{2} \sin ^{2} \theta\left(2 \Omega^{2} \alpha_{4}-\alpha_{6} \Omega+2 \Gamma^{2} \alpha_{4}+\alpha_{5} \Gamma\right)}{\rho \cos ^{3} \theta\left(\Omega^{2}+\Gamma^{2}\right)}, \operatorname{Im}\left\{\varepsilon_{z z}\right\}=\frac{\operatorname{Im}\{a\}}{\cos ^{2} \theta}+(\pi / 2)^{1 / 2} \frac{\eta^{2} \sin ^{2} \theta\left(\alpha_{6} \Gamma+\alpha_{5} \Omega-2 \alpha_{3} \Gamma^{2}-2 \alpha_{3} \Omega^{2}\right)}{\rho \cos ^{3} \theta\left(\Omega^{2}+\Gamma^{2}\right)}, \\
\alpha_{1}= & \sum_{s=-\infty}^{\infty}\left[\frac{A_{s-1}+A_{s+1}}{2}-A_{s}\right] \operatorname{Re} W, \alpha_{2}=\sum_{s=-\infty}^{\infty}\left[\frac{A_{s-1}+A_{s+1}}{2}-A_{s}\right] \operatorname{Im} W, \alpha_{3}=\sum_{s=-\infty}^{\infty} \frac{A_{s-1}-A_{s+1}}{2} \operatorname{ReW,}
\end{aligned}
$$


V. G. Ledenev et al.: High-frequency waves in the solar coronal plasma, Online Material p 3

$\alpha_{4}=\sum_{s=-\infty}^{\infty} \frac{A_{s-1}-A_{s+1}}{2} \operatorname{Im} W, \alpha_{5}=\sum_{s=-\infty}^{\infty} s \frac{A_{s-1}-A_{s+1}}{2} \operatorname{Re} W, \alpha_{6}=\sum_{s=-\infty}^{\infty} s \frac{A_{s-1}-A_{s+1}}{2} \operatorname{Im} W$,

$\alpha_{7}=\sum_{s=-\infty}^{\infty} s\left[\frac{A_{s-1}+A_{s+1}}{2}-A_{s}\right] \operatorname{Re} W, \alpha_{8}=\sum_{s=-\infty}^{\infty} s\left[\frac{A_{s-1}+A_{s+1}}{2}-A_{s}\right] \operatorname{Im} W$.

The function $W \rightarrow W\left(\frac{\Omega-s}{\rho \sqrt{2} \cos \theta}+\mathrm{i} \frac{\Gamma}{\rho \sqrt{2} \cos \theta}\right)$ has the form (Abramovitz \& Stegun 1964):

$W\left(x_{0}+\mathrm{i} y_{0}\right)=\operatorname{Re} W\left(x_{0}+\mathrm{i} y_{0}\right)+\mathrm{i} \operatorname{Im} W\left(x_{0}+i y_{0}\right)$,

$\operatorname{Re} W\left(x_{0}+\mathrm{i} y_{0}\right)=\left[\exp \left(y_{0}^{2}-x_{0}^{2}\right)\right]\left[\cos \left(2 x_{0} y_{0}\right)-\cos \left(2 x_{0} y_{0}\right) \operatorname{erf}\left(y_{0}\right)+\frac{2}{\sqrt{\pi}}\left[\exp \left(-y_{0}^{2}\right) \int_{0}^{x_{0}} \partial x\left[\exp \left(x^{2}\right) \sin \left[2\left(x_{0}-x\right) y_{0}\right]\right.\right.\right.$,

$\operatorname{Im} W\left(x_{0}+\mathrm{i} y_{0}\right)=\left[\exp \left(y_{0}^{2}-x_{0}^{2}\right)\right]\left[-\sin \left(2 x_{0} y_{0}\right)+\sin \left(2 x_{0} y_{0}\right) \operatorname{erf}\left(y_{0}\right)+\frac{2}{\sqrt{\pi}}\left[\exp \left(-y_{0}^{2}\right) \int_{0}^{x_{0}} \partial x\left[\exp \left(x^{2}\right) \cos \left[2\left(x_{0}-x\right) y_{0}\right]\right.\right.\right.$.

In the region $\left(x_{0}^{2}+y_{0}^{2}\right)^{1 / 2} \gg 1$, the real and imaginary parts of the function $W$ have the form (Abramovitz \& Stegun 1964):

$$
\begin{aligned}
\sqrt{\pi} \operatorname{Re} W\left(x_{0}+i y_{0}\right) \approx & \frac{y_{0}}{x_{0}^{2}+y_{0}^{2}}-\frac{y_{0}^{3}-3 y_{0} x_{0}^{2}}{2\left(x_{0}^{2}+y_{0}^{2}\right)^{3}}+\frac{3\left(y_{0}^{5}-10 x_{0}^{2} y_{0}^{3}+5 x_{0}^{4} y_{0}\right)}{4\left(x_{0}^{2}+y_{0}^{2}\right)^{5}} \\
& -\frac{15\left[\left(y_{0}^{2}-x_{0}^{2}\right)\left(y_{0}^{5}-10 x_{0}^{2} y_{0}^{3}+5 x_{0}^{4} y_{0}\right)-2 y_{0} x_{0}\left(x_{0}^{5}+5 x_{0} y_{0}^{4}-10 y_{0}^{2} x_{0}^{3}\right)\right]}{8\left(x_{0}^{2}+y_{0}^{2}\right)^{7}},
\end{aligned}
$$

$\sqrt{\pi} \operatorname{Im} W\left(x_{0}+i y_{0}\right) \approx \frac{x_{0}}{x_{0}^{2}+y_{0}^{2}}-\frac{3 x_{0} y_{0}^{2}-x_{0}^{3}}{2\left(x_{0}^{2}+y_{0}^{2}\right)^{3}}+\frac{3\left(x_{0}^{5}+5 x_{0} y_{0}^{4}-10 y_{0}^{2} x_{0}^{3}\right)}{4\left(x_{0}^{2}+y_{0}^{2}\right)^{5}}$

$$
-\frac{15\left[\left(y_{0}^{2}-x_{0}^{2}\right)\left(x_{0}^{5}+5 x_{0} y_{0}^{4}-10 y_{0}^{2} x_{0}^{3}\right)+2 x_{0} y_{0}\left(y_{0}^{5}-10 x_{0}^{2} y_{0}^{3}+5 x_{0}^{4} y_{0}\right]\right.}{8\left(x_{0}^{2}+y_{0}^{2}\right)^{7}} \text {. }
$$

The system of Eqs. (A.1) describes waves in a hot magnetized plasma with a Maxwellian distribution function. 